\title{
Li-Ion Batteries State-of-Charge Estimation Using Deep LSTM at Various Battery Specifications and Discharge Cycles
}

\author{
Kei Long Wong*, Michael Bosello ${ }^{\dagger}$, Rita Tse*, Carlo Falcomer ${ }^{\dagger}$, Claudio Rossi ${ }^{\ddagger}$, Giovanni Pau ${ }^{\dagger, \#}$ \\ *School of Applied Sciences, Macao Polytechnic Institute, Macao SAR \\ ${ }^{\dagger}$ DISI -Dept. of Computer Science and Engineering, University of Bologna, Italy \\ *DEI -Dept. of Electrical, Electronic, and Information Engineering, University of Bologna, Italy \\ \# UCLA Computer Science, Los Angeles, USA
}

\begin{abstract}
Lithium-ion battery technologies play a key role in transforming the economy reducing its dependency on fossil fuels. Transportation, manufacturing, and services are being electrified. The European Commission predicts that in Europe everything that can be electrified will be electrified within a decade. The ability to accurate state of charge (SOC) estimation is crucial to ensure the safety of the operation of battery-powered electric devices and to guide users taking behaviors that can extend battery life and re-usability. In this paper, we investigate how machine learning models can predict the SOC of cylindrical Li-Ion batteries considering a variety of cells under different charge-discharge cycles.
\end{abstract}

\section{CCS CONCEPTS}

- Computing methodologies $\rightarrow$ Neural networks; Supervised learning by regression.

\section{KEYWORDS}

Lithium-ion battery, long short-term memory, recurrent neural network, state of charge estimation

\section{ACM Reference Format:}

Kei Long Wong*, Michael Bosello ${ }^{\dagger}$, Rita Tse*, Carlo Falcomer ${ }^{\dagger}$, Claudio Rossi $^{\ddagger}$, Giovanni Pau ${ }^{\dagger}, \#$. 2021. Li-Ion Batteries State-of-Charge Estimation Using Deep LSTM at Various Battery Specifications and Discharge Cycles. In Conference on Information Technology for Social Good (GoodIT'21), September 9-11, 2021, Roma, Italy. ACM, New York, NY, USA, 6 pages. https://doi.org/ $10.1145 / 3462203.3475878$

\section{INTRODUCTION}

The transition from fossil fuel to green energy is well known as the desired change in our society. To reduce the emission of Carbon dioxide $\left(\mathrm{CO}_{2}\right)$ from conventional transportation, the development of Electric Vehicles (EV) is growing quickly. Battery technology will be one of the most important key enablers for the green energy transition.

Lithium-ion batteries have been widely used in electric vehicles. It is projected that the global EV stock will expand to 140 million by

Permission to make digital or hard copies of all or part of this work for personal or classroom use is granted without fee provided that copies are not made or distributed for profit or commercial advantage and that copies bear this notice and the full citation on the first page. Copyrights for components of this work owned by others than ACM must be honored. Abstracting with credit is permitted. To copy otherwise, or republish, to post on servers or to redistribute to lists, requires prior specific permission and/or a fee. Request permissions from permissions@acm.org.

GoodIT '21, September 9-11, 2021, Roma, Italy

(C) 2021 Association for Computing Machinery.

ACM ISBN 978-1-4503-8478-0/21/09.

https://doi.org/10.1145/3462203.3475878
2030 [1]. Lithium-ion (Li-ion) battery is the most popular adopted power supply of EV due to its high energy density, long lifespan, lightweight, and low self-discharge rate [19]. Several factors could affect the performance and safety of Li-ion battery such as ambient temperature, over-charge, or over-discharge [20, 21]. A misuse of the battery can lead to a shorter battery life. To overcome these issues, Battery Management Systems (BMS) are applied to ensure the reliability and stability of the usage of Li-ion batteries.

One important parameter for the BMS battery health management is the battery State Of Charge (SOC) estimation which helps to prevent the battery from over-charge and over-discharge [10, 28]. SOC indicates the amount of available charge in the battery which can be represented by a value in percentage. This value is intended to remain between $0 \%$ and $100 \%$, although it is possible to violate these limits in an over-discharge or over-charge situation [24]. The battery itself does not directly provide information on its SOC value. The measurement of SOC value is complex and error-prone due to the indirect estimation and the non-linear nature of electrochemical reactions in the battery. Relevant information such as the measured discharge current, voltage, and ambient temperature can be used to measure the SOC indirectly [5].

Incorrect measurement of SOC could lead to unstable EV performance and even shorten the battery life, therefore, reducing the environmental benefits of electrification. In general, the SOC estimation techniques studied in the literature can be divided into three categories: direct methods, model-based methods, and datadriven methods [25]. The direct methods look for the relationship between SOC and the physical battery characteristic parameters. The SOC value can be estimated according to the observed parameters[24],[25], [22]. The model-based SOC estimation methods mainly focus on modeling the chemical and electrical properties of the battery. Commonly, the model-based methods are used in collaboration with adaptive filters such as Kalman filter, H-infinity filter, and Particle filter, etc [25], [23]. Model-based methods require a comprehensive understanding of the electrochemical properties in the battery domain and cannot be used for SOC forecast[12][3].

This work proposes a data-driven approach for SOC estimation based on Deep Learning techniques. Deep learning, which can approximate non-linear functions, is a widely adopted data-driven method to tackle the battery SOC estimation problem [27]. Given a sufficient amount of training data and an appropriate configuration, the SOC value can be predicted accurately without the need for a sophisticated electrochemical model. Different types of neural networks (NNs) such as Convolutional Neural Networks (CNNs) and Recurrent Neural Networks (RNNs) have been studied in the literature to solve various problems of different nature [8, 14]. Among 
them, RNNs are designed to handle sequential data, and they have been well studied in the domain of speech recognition and natural language processing with successful outcomes [9, 30]. However, RNNs struggle to handle long-term dependencies as long time series could cause exploding/vanishing gradient during the training phase. To tackle this problem, Hochreiter and Schmidhuber [11] proposed the use of RNNs with Long Short-Term Memory (LSTM) cells which can correlate a long-range of precedent information.

In the literature, various applications of LSTM for SOC estimation have been proposed. Chemali, Kollmeyer, Preindl, Ahmed, and Emadi [4] showcased the ability of LSTM for SOC estimation. They used a dataset composed of discharge cycles obtained from a Li-ion battery cell with 2.9Ah nominal capacity [17] and collected through laboratory testing under different driving profiles. The proposed model was validated against various ambient temperatures with accurate estimation results achieved. Similarly, Yang, Song, Xu, and Tsui [29] proposed the use of a deep LSTM network with data collected from an experiment on a 1.1Ah nominal capacity battery cell. In [26], a neural network combining CNN and LSTM layers was proposed for battery SOC estimation. The research result has shown that the $\mathrm{CNN}$ part helps to extract the spatial features from the input data (voltage, current, and temperature) while the LSTM layers explore the correlation of current SOC and historical input data. Last but not least, the use of an LSTM encoder-decoder algorithm was proposed by Cui, Yong, Kim, Hong, and Joe [7] with accurate estimation result against both room temperature and various temperature conditions. The proposed model was trained and tested on 2.0Ah nominal capacity Li-ion battery cell data featuring various drive cycles.

Although the neural network data-driven approaches for SOC estimation are widely studied most of the literature mainly focuses on datasets containing only one particular battery model or setup. This study uses two different Li-ion cell datasets. The first one is original and it has been collected by the University of Bologna (UNIBO), namely the 'UNIBO Powertools Dataset'. The second one is public, the LG $18650 \mathrm{HG} 2 \mathrm{Li}$-ion battery data [18]. The use of deep LSTM networks is proposed to perform SOC estimation. Due to the heterogeneity of the data collection process and the sampling rate of the two datasets, two deep LSTM models with different setups are employed in this research. The deep networks are tested on Li-ion battery cells with different nominal capacities, specifications, and brands; the discharge cycles are produced by both constant current discharge and several dynamic driving profiles (such as the Urban dynamometer driving schedule (UDDS) [2]).

The rest of this paper is organized as follows. The employed battery datasets are introduced in section 2 . Then, section 3 explains the proposed deep LSTM models. In section 4, the results of the experiments are presented. Finally, section 5 concludes this paper.

\section{LI-ION BATTERY DATASETS}

In this paper, two Li-ion battery datasets - one original and one public - with different features are used. The UNIBO Powertools Dataset is presented here for the first time, and it is available here ${ }^{1}$ Only the discharge cycles are used in the experiments. The two datasets are briefly introduced in the following sections.

\footnotetext{
${ }^{1}$ https://doi.org/10.17632/n6xg5fzsbv.1
}

Table 1: UNIBO Powertools Dataset summary

\begin{tabular}{ccc}
\hline Test type & Nominal capacity & Cell amount \\
\hline Standard & $4.0 \mathrm{Ah}$ & 2 \\
\cline { 2 - 3 } & $3.0 \mathrm{Ah}$ & 4 \\
\cline { 2 - 3 } & $2.85 \mathrm{Ah}$ & 4 \\
\cline { 2 - 3 } & $2.0 \mathrm{Ah}$ & 6 \\
\hline High current & $3.0 \mathrm{Ah}$ & 3 \\
\cline { 2 - 3 } & $2.85 \mathrm{Ah}$ & 2 \\
\hline Preconditioned & $3.0 \mathrm{Ah}$ & 5 \\
\hline
\end{tabular}

\subsection{UNIBO Powertools Dataset}

The UNIBO Powertools Dataset has been collected in a laboratory test by an Italian Equipment producer. The cycling experiments are designed to analyze different cells intended for use in various cleaning equipment such as vacuum and automated floor cleaners. The vast dataset is composed of 27 batteries, and it is summarized in Table 1. The main features of the dataset are: (1) the use of batteries from different manufacturers, (2) cells with several nominal capacities, (3) cycling is performed until the cell's end of life and thus data regarding the cell at different life stages are produced, which is useful to assess how SOC is affected by the cell's age and State of Health ( $\mathrm{SOH}$ ) as well as to validate the capability of the proposed model on estimating SOC under different health status. Three types of tests have been conducted. (I) The standard test, where the battery was discharged at $5 \mathrm{~A}$ current in main cycles. (II), the high current test, where the battery was discharged at $8 \mathrm{~A}$ current in main cycles. (III), the preconditioned test, where the battery cells are stored at $45^{\circ} \mathrm{C}$ environment for 90 days before conducting the test.

During discharge, the sampling period is 10 seconds. The experiments were conducted using the following procedure:

(1) Charge cycle: Constant Current-Constant Voltage (CC-CV) at $1.8 \mathrm{~A}$ and $4.2 \mathrm{~V}(100 \mathrm{~mA}$ cut-off)

(2) Discharge cycle: Constant Current until cut-off voltage (2.5V)

(3) Repeat steps 1 and 2 (main cycle) 100 times

(4) Capacity measurement: charge CC-CV $1 \mathrm{~A} 4.2 \mathrm{~V}$ (100mA cutoff) and discharge CC $0.1 \mathrm{~A} 2.5 \mathrm{~V}$

(5) Repeat the above steps until the end of life of the battery cell

\subsection{LG 18650HG2 Li-ion Battery Data}

The public LG 18650HG2 Li-ion Battery Dataset, published by Kollmeyer, Vidal, Naguib, and Skells [18], was obtained from Mendeley data. In the dataset, a series of tests were performed under six different temperatures. The battery was charged at $1 \mathrm{C}$ rate to $4.2 \mathrm{~V}$ with $50 \mathrm{~mA}$ cut off before each discharge test. The values measured in the discharge cycles are captured at 0.1 seconds sampling period. Different drive cycles such as UDDS, LA92, and US06, as well as mixes of them, are applied in the discharge tests. In this paper, the discharge cycles with temperature of $0^{\circ} \mathrm{C}, 10^{\circ} \mathrm{C}$ and $25^{\circ} \mathrm{C}$ were used for training and testing the proposed model.

\section{METHODOLOGY}

In this section, the basic theories of RNN and LSTM are introduced and the two proposed deep LSTM models are briefly introduced. Then, the normalization method used to scale the input data is reviewed. Lastly, the model's configuration is discussed. 


\subsection{Recurrent Neural Networks Primer}

Recurrent neural networks are a class of neural networks that allows the information to persist over time. Different from the feedforward neural networks that are acyclic directed graphs, RNNs have connections within layers forming cyclic directed graphs. This empowers neural networks to have a state, and thus memory. The information from the previous state is utilized as input along with the current time step. It is useful for sequential data prediction as relationships between current and past information are considered. An example of the architecture of an RNN for SOC estimation unfolded in time, is depicted in Fig. 1. The input vector at the time step $t$ contains battery parameters such as voltage, current, and temperature, and it is denoted as Input $t_{t} . h_{t}$ represents the hidden state at time step $t$, while the output SOC value at time step $t$ is denoted as $S O C_{t}$. Fig. 1 demonstrates a common approach for timeseries called many-to-many, where multiple input steps are fed to the network with one prediction made at each step. Whereas, there are other approaches such as the many-to-one and one-to-many, where in the first case multiple time-steps are fed with one output produced, and in the second case one input is used to produce multiple time-steps. As the two battery datasets have very different sampling frequencies, we used the many-to-many approach for the first model (low-frequency sampling) while in the second one (high-frequency sampling) we used the many-to-one approach.

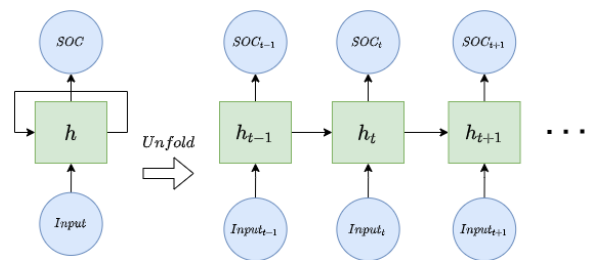

Figure 1: RNN architecture for SOC estimation unfolded in time

\subsection{Long Short-Term Memory Primer}

The long short-term memory is a type of RNN which is widely used to learn long-term dependencies without experiencing the exploding and vanishing gradient problems. The forward pass of an LSTM cell can be defined by the following steps. In the equations, $f_{t}, i_{t}, o_{t}$ are the forget-gate, input-gate, output-gate; $c_{t}$ and $h_{t}$ are the cell state and hidden state at time step $t$ respectively; $\sigma$ is the sigmoid function; $\odot$ is the Hadamard product; $W$ denotes the weight matrix; $x_{t}$ is the input vector at time step $t$ and $b$ is the bias.

The first step in the LSTM cell is to determine what information will be forgotten from the cell state $c_{t-1}$. The forget-gate uses a sigmoid function, in which outputs are always between 0 and 1 . The result represents therefore how much should be forgotten, with 0 and 1 representing respectively discarding everything or keeping everything from the previous cell's state. As shown in the equations, the decisions of gates are based on the current input and hidden state as well as on the network's weights and biases.

$$
f_{t}=\sigma\left(W_{x}^{f} x_{t}+W_{h}^{f} h_{t-1}+b^{f}\right)
$$

The second step determines whether the information will be stored in the cell state. There are two parts in the second step. Firstly, the input-gate with sigmoid output determines to what extent the value will be remembered. Secondly, the tanh layer generates the new value $\tilde{c}_{t}$ that is multiplied by the sigmoid output and then added to the cell state.

$$
\begin{aligned}
& i_{t}=\sigma\left(W_{x}^{i} x_{t}+W_{h}^{i} h_{t-1}+b^{i}\right) \\
& \tilde{c}_{t}=\tanh \left(W_{x}^{c} x_{t}+W_{h}^{c} h_{t-1}+b^{c}\right)
\end{aligned}
$$

The cell state $c_{t}$ is then update combining the previous cell state $c_{t-1}$ with new value $\tilde{c}_{t}$ as mentioned above. The forget-gate $f_{t}$ and input-gate $i_{t}$ determine whether the values should be discarded or remembered.

$$
c_{t}=f_{t} \odot c_{t-1}+i_{t} \odot \tilde{c}_{t}
$$

In the last step, the output-gate with the sigmoid function decides which part of the cell state is propagated to the hidden state $h_{t}$. In the hidden state, the cell state $c_{t}$ is passed via tanh and multiplied by the output-gate to keep only the desired output.

$$
\begin{aligned}
& o_{t}=\sigma\left(W_{x}^{o} x_{t}+W_{h}^{o} h_{t-1}+b^{o}\right) \\
& h_{t}=o_{t} \odot \tanh \left(c_{t}\right)
\end{aligned}
$$

\subsection{Proposed LSTM Approach}

There are two deep LSTM models proposed in this paper, one for each dataset, as they have very different cycle lengths. Scaled exponential linear units (SELU) [16] activation function is used in all the LSTM cells and hidden dense layers. In the output layer, the linear activation function is applied to produce the final SOC value.

The first model is used for the UNIBO dataset. It is a deep neural network with three LSTM layers followed by two dense layers to map the learned states to desired SOC output. The number of cells of each LSTM layer is 256, 256, and 128 respectively. Fig. 2 illustrates the architecture of the first proposed model. The first layer is the input layer with battery parameters including voltage $V$, current $I$, and temperature $T$ at each step $t$. Since it is a deep LSTM network, each LSTM layer returns a sequence which means that each step is propagated to the next layer. Here, we adopted the many-tomany approach, the SOC value is therefore estimated at every step. The input time series fed to the deep LSTM network is defined as $\left[\right.$ Input $_{t 0}$, Input $_{t 1}, \ldots$ Input $\left._{t n}\right]$, where $n$ is the number of steps in the entire discharge cycle, and Input $=\left[V_{t}, I_{t}, T_{t}\right]$ represents voltage, current and temperature at each time step respectively. Although the entire discharge cycle is fed to the network, only the part that precedes the step under examination is available as input for SOC estimation, i.e., the hidden state from previous steps $t-1$ and the current input at step $t$ are used to estimate the output at step $t$.

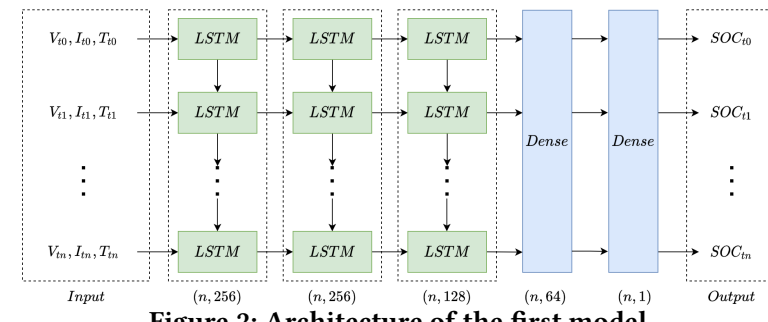

Figure 2: Architecture of the first model 
The second model is used for the LG $18650 \mathrm{HG} 2 \mathrm{Li}$-ion battery dataset. The model is composed of two LSTM layers followed by three dense layers. The number of cells of both LSTM layers is 256 . Fig. 3 shows the architecture of the second proposed model. Since the second dataset contains more steps in one discharge cycle due to its higher sampling rate ( 0.1 seconds sampling time), the manyto-one approach is more appropriate. In this case, for each $n$ step as input, one output is returned. In the implementation, we used 300 , 500 , and 700 as the number of steps. For example, given input steps $\left[\right.$ Input $_{t 0}$, Input $_{t 1}, \ldots$ Input $\left._{t 500}\right]$, the model should estimate the SOC value at step 500 .

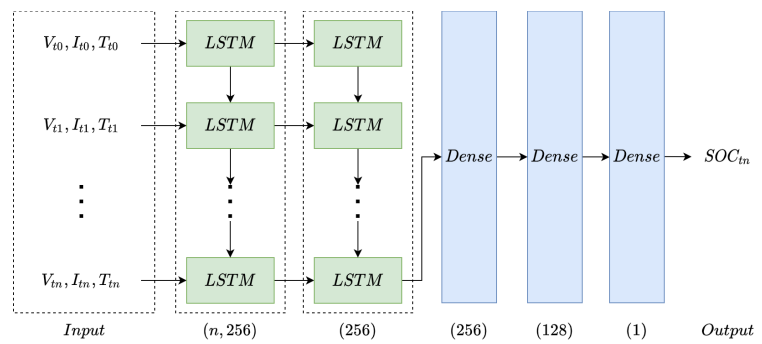

Figure 3: Architecture of the second model

\subsection{Data Normalization}

Since the input features have different ranges, such as the temperature has much higher values than voltage and current, the trained model could give more importance to this feature over the others due to its larger value. To avoid this problem, the minimummaximum normalization method is used to scale all input features into the same common scale.

\subsection{Model Training}

The proposed models are implemented by using the Keras library [6]. The Adam algorithm [15] is chosen as the optimizer to update the network weights and biases with the learning rate configured as 0.00001 . All proposed models are trained for 1000 epochs, but the training process would stop earlier if there is no further improvement of validation loss within 50 epochs. The Huber loss [13] is used as the loss function. Its peculiarity is that it can be quadratic or linear depending on the error value.

\section{RESULTS AND DISCUSSION}

The proposed deep LSTM models are trained and tested using the two aforementioned datasets. The model performance against each dataset is discussed in this section. The source code of the model implementation and results are available here ${ }^{2}$.

Root Mean Square Error (RMSE) and Mean absolute error (MAE) are used to evaluate the proposed models. The Mean Square Error (MSE) is the sum of squared distances between the target and predicted variables divided by the number of samples. The RMSE is the square root of the MSE which scales the output value to the same scale as MAE. It is more sensitive to outliers as it penalizes the model by squaring the error. The MAE on the other hand is more robust to outliers as the error is not squared. MAE is an L1 loss

\footnotetext{
${ }^{2}$ https://github.com/KeiLongW/battery-state-estimation
}

Table 2: UNIBO dataset tests performance

\begin{tabular}{cccc}
\hline Test type & Nominal capacity & MAE & RMSE \\
\hline Standard & $4.0 \mathrm{Ah}$ & $2.68 \%$ & $3.42 \%$ \\
\cline { 2 - 4 } & $3.0 \mathrm{Ah}$ & $0.52 \%$ & $0.73 \%$ \\
\cline { 2 - 4 } & $2.85 \mathrm{Ah}$ & $0.31 \%$ & $0.39 \%$ \\
\cline { 2 - 4 } & $2.0 \mathrm{Ah}$ & $0.59 \%$ & $0.80 \%$ \\
\hline High current & $3.0 \mathrm{Ah}$ & $0.46 \%$ & $0.61 \%$ \\
\cline { 2 - 4 } & $2.85 \mathrm{Ah}$ & $2.13 \%$ & $3.24 \%$ \\
\hline Preconditioned & $3.0 \mathrm{Ah}$ & $0.47 \%$ & $0.66 \%$ \\
\hline
\end{tabular}

function that calculates the sum of the absolute difference between the target and predicted variables. The MAE is more suitable for problems where the training data present outliers.

\subsection{UNIBO Powertools Dataset}

In the UNIBO dataset tests, the performance of the proposed model is evaluated over constant current discharge. The proposed model for this dataset was trained with a total of 7738 discharging cycles as the training set. One cell for each group of test types (standard, high current, pre-conditioned) and nominal capacity was extracted as testing data for evaluation purposes. The testing data is isolated from training data so that it is unseen during the training process. The overall MAE and RMSE on all testing data are $0.69 \%$ and $1.34 \%$ respectively.

To further investigate the performance of the proposed model, Table 2 shows the performance of each test type. The evaluation of standard test type with 4.0Ah nominal capacity and high current test type with $2.85 \mathrm{Ah}$ nominal capacity has the worst performance. This is expected as the dataset contains only two cell tests of the kind, resulting in one cell used for training and one for testing. Whereas, in the other test types with sufficient data the model can achieve accurate results with RMSE lower than $1 \%$.

The examples of SOC estimation results of the proposed model on the standard, high current, and preconditioned test types are shown in Fig. 4, Fig. 5, and Fig. 6 respectively. The first and the last discharge cycles within the entire test of each battery cell are presented to demonstrate the SOC estimation performance under different health statuses. All results show the discharge process of SOC being discharged from $100 \%$ to $0 \%$. The $\mathrm{x}$-axis represents the discharge steps over the whole discharging cycle and the $y-$ axis represents the SOC value at each step. The black line is the actual observed SOC value during the discharge process and the red dashed line is the SOC value estimated by the proposed model.

The model estimates the SOC of the 3.0Ah nominal capacity cells correctly and without large fluctuation in each of the three test types. Furthermore, the estimations of standard test types with 2.0Ah and 2.85Ah nominal capacity are accurate too. SOC in both the first and last cycle are estimated accurately which suggests that the proposed model is capable to estimate SOC under different battery health statuses. In addition, good performance is achieved from the preconditioned test type which demonstrates that the storage temperature before testing does not affect the battery discharging behavior significantly in terms of SOC estimation. On the other hand, there are some errors during the ending steps of standard 4.0Ah nominal capacity and high current $2.85 \mathrm{Ah}$ nominal capacity battery cell cycles. It is acceptable as there is only one training example of that kind of setup. 


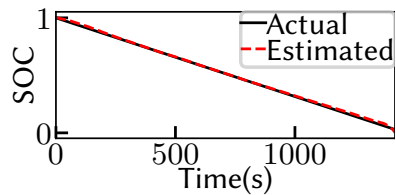

(a) Standard 2.0Ah (first cycle)

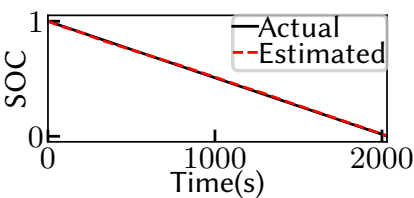

(c) Standard 2.85Ah (first cycle)

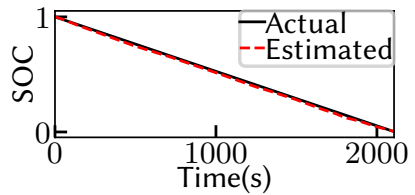

(e) Standard 3.0Ah (first cycle)

(g) Standard 4.0Ah (first cycle)

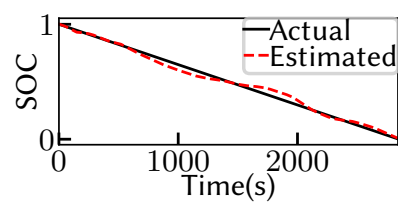

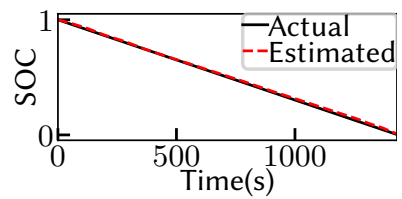

(b) Standard 2.0Ah (last cycle)

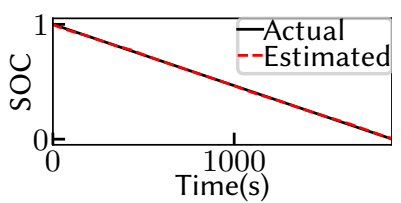

(d) Standard 2.85Ah (last cycle)

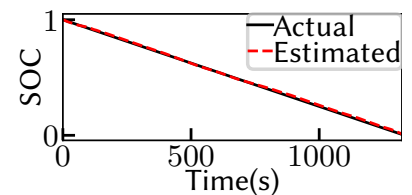

(f) Standard 3.0Ah (last cycle)

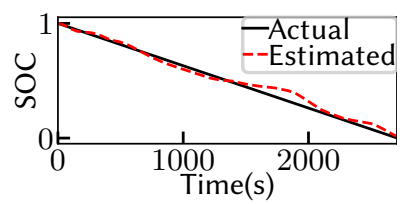

(h) Standard 4.0Ah (last cycle)
Figure 4: UNIBO dataset SOC estimation results (standard)

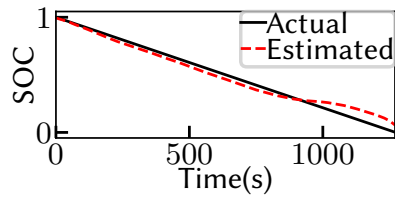

(a) High current $2.85 \mathrm{Ah}$ (first cycle)

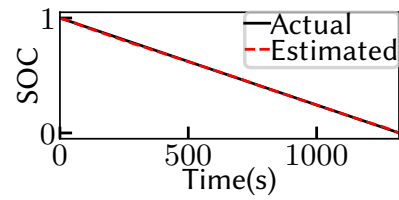

(c) High current $3.0 \mathrm{Ah}$ (first cycle)

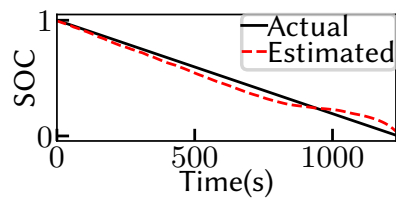

(b) High current $2.85 \mathrm{Ah}$ (last cycle)

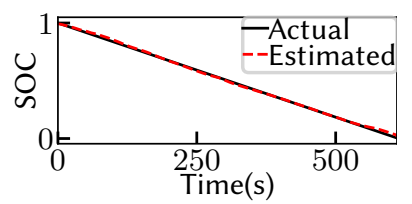

(d) High current $3.0 \mathrm{Ah}$ (last cycle)
Figure 5: UNIBO dataset SOC estimation results (high current)

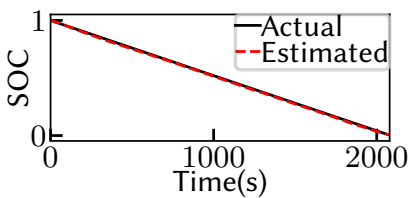

(a) Preconditioned 3.0Ah (first cycle)

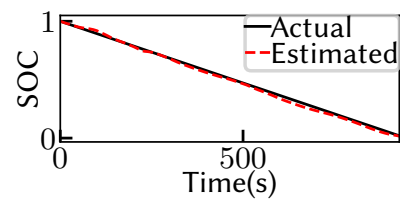

(b) Preconditioned 3.0Ah (last cycle)
Figure 6: UNIBO dataset SOC estimation results (preconditioned)
Table 3: LG 18650HG2 data tests performance

\begin{tabular}{crrrrrr}
\hline \multirow{2}{*}{ Temp. $\left({ }^{\circ} \mathrm{C}\right)$} & \multicolumn{2}{c}{300 Steps } & \multicolumn{2}{c}{500 Steps } & \multicolumn{2}{c}{ 700 Steps } \\
& MAE & RMSE & MAE & RMSE & MAE & RMSE \\
\hline 0 & $1.69 \%$ & $2.27 \%$ & $\mathbf{1 . 4 7 \%}$ & $\mathbf{2 . 2 3 \%}$ & $1.65 \%$ & $2.60 \%$ \\
\hline 10 & $1.61 \%$ & $2.12 \%$ & $\mathbf{1 . 5 7 \%}$ & $\mathbf{2 . 1 2 \%}$ & $2.22 \%$ & $2.89 \%$ \\
\hline 25 & $\mathbf{1 . 1 7 \%}$ & $\mathbf{1 . 5 7 \%}$ & $1.59 \%$ & $2.02 \%$ & $1.92 \%$ & $2.64 \%$ \\
\hline
\end{tabular}

\subsection{LG 18650HG2 Li-ion Battery Data}

In the LG $18650 \mathrm{HG} 2 \mathrm{Li}$-ion battery dataset, the performance of the proposed model under dynamic discharge current is evaluated. Six mixed driving cycles for each of three different temperatures $0^{\circ} \mathrm{C}$, $10^{\circ} \mathrm{C}$, and $25^{\circ} \mathrm{C}$ were used as training set. We have also tested three different time series lengths, with a number of steps of 300,500 , and 700 , which are approximately equal to 30 seconds, 50 seconds, and 70 seconds depth in time respectively. The test set was composed of a UDDS, an LA92, and a US06 driving cycle plus one mixed driving cycle for each of the three different temperatures available in the dataset.

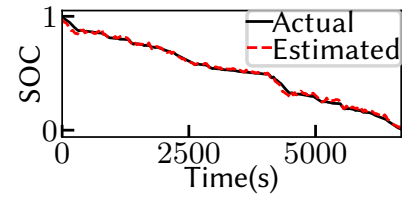

(a) $25^{\circ} \mathrm{C}$ (300 steps)

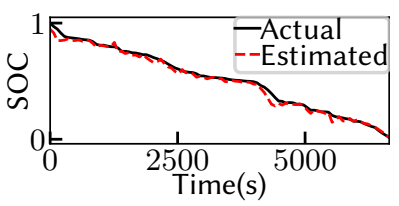

(c) $25^{\circ} \mathrm{C}$ (700 steps)

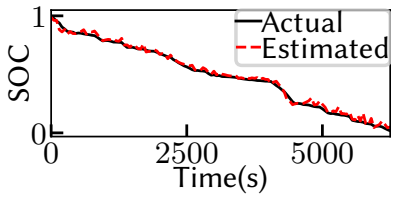

(e) $10^{\circ} \mathrm{C}$ (500 steps)

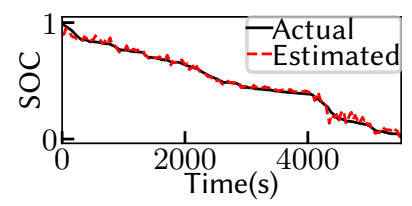

(g) $0^{\circ} \mathrm{C}$ (300 steps)

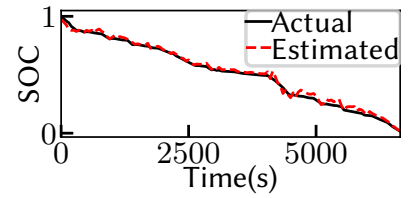

(b) $25^{\circ} \mathrm{C}$ (500 steps)

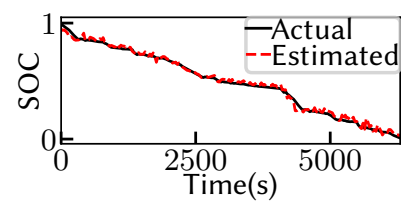

(d) $10^{\circ} \mathrm{C}$ (300 steps)

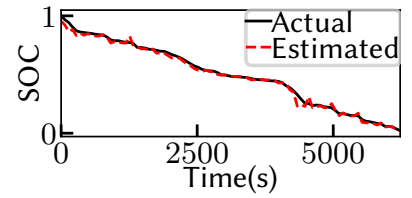

(f) $10^{\circ} \mathrm{C}$ (700 steps)

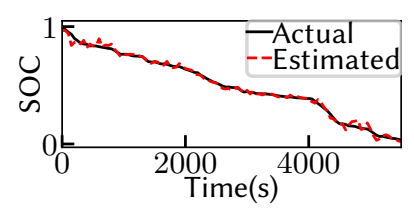

(h) $0^{\circ} \mathrm{C}$ (500 steps)

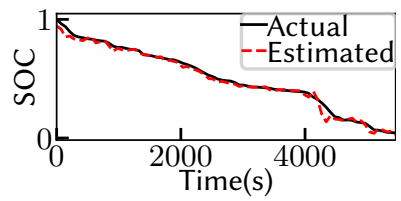

(i) $0^{\circ} \mathrm{C}$ (700 steps)

Figure 7: LG 18650HG data SOC estimation results (mixed cycles) 
The MAE and RMSE achieved by the 300 steps model are $1.47 \%$ and $1.99 \%$. The 500 steps one reached an MAE and RMSE of $1.54 \%$ and $2.12 \%$. The 700 steps model achieved $1.94 \%$ MAE and $2.72 \%$ RMSE. All the aforementioned results were tested with testing data under all temperatures. The model performance under each temperature with different input lengths is listed in Table 3. Among all the configurations, the best performance is achieved from testing data under $25^{\circ} \mathrm{C}$ temperature with 300 steps in input, which demonstrates that the battery operates most stably under room temperature. The model is able to learn the battery behavior under room temperature through the provided driving cycles without the need for a long history. While, under $10^{\circ} \mathrm{C}$ and $0^{\circ} \mathrm{C}$ temperatures, better performance is gained from the 500 input model. This indicates that increasing input steps could help to improve the estimation result under temperatures that are lower than room temperature. However, the worst results are from the 700 input steps which suggest that the increment of input steps must be selected carefully for the many-to-one approach as an inappropriate increment of input steps could result in performance degradation. The SOC estimation results on the mixed driving cycles under $0^{\circ} \mathrm{C}$, $10^{\circ} \mathrm{C}$ and $25^{\circ} \mathrm{C}$ temperatures are displayed in Fig. 7. The estimation results under the three temperatures are competitive and without significant errors. Still, errors can be seen from the ending steps in mixed cycles under $0^{\circ} \mathrm{C}$ temperature due to their more dynamic discharge pattern.

\section{FINAL REMARKS}

In this paper, a deep LSTM NN is proposed to estimate SOC over two different Li-ion battery datasets. Discharge cycles with both constant and dynamic current under various ambient temperatures are used to train and test the proposed models. The evaluation results show that the proposed models can learn the battery dynamic behavior during discharge. Battery SOC can be estimated accurately by using the measured voltage, current, and temperature values, with $1.34 \%$ and $1.99 \%$ RMSE in constant current and dynamic current discharge cycle respectively. We have also shown how the proposed estimation is robust w.r.t. different State of Health statuses. The $\mathrm{SOH}$ is another important parameter for battery management. As future work, we suggest using deep LSTM networks for $\mathrm{SOH}$ estimation as we believe it can be effective as well.

\section{REFERENCES}

[1] International Energy Agency. 2020. Global EV Outlook 2020. OECD Publishing, Paris. 276 pages. https://doi.org/10.1787/d394399e-en

[2] United States Environmental Protection Agency. 2020. EPA Urban Dynamometer Driving Schedule (UDDS). https://www.epa.gov/emission-standards-referenceguide/epa-urban-dynamometer-driving-schedule-udds

[3] Christian Campestrini, Thomas Heil, Stephan Kosch, and Andreas Jossen. 2016 A comparative study and review of different Kalman filters by applying an enhanced validation method. Journal of Energy Storage 8 (2016), 142-159. https: //doi.org/10.1016/j.est.2016.10.004

[4] Ephrem Chemali, Phillip J. Kollmeyer, Matthias Preindl, Ryan Ahmed, and Ali Emadi. 2018. Long Short-Term Memory Networks for Accurate State-of-Charge Estimation of Li-ion Batteries. IEEE Transactions on Industrial Electronics 65, 8 (2018), 6730-6739. https://doi.org/10.1109/TIE.2017.2787586

[5] K. W. E. Cheng, B. P. Divakar, Hongjie Wu, Kai Ding, and Ho Fai Ho. 2011. BatteryManagement System (BMS) and SOC Development for Electrical Vehicles. IEEE Transactions on Vehicular Technology 60, 1 (2011), 76-88. https://doi.org/10.1109/ TVT.2010.2089647

[6] François Chollet and Others. 2015. Keras. https://keras.io.

[7] Shengmin Cui, Xiaowa Yong, Sanghwan Kim, Seokjoon Hong, and Inwhee Joe. 2020. An LSTM-Based Encoder-Decoder Model for State-of-Charge Estimation of Lithium-Ion Batteries. In Intelligent Algorithms in Software Engineering, Radek Silhavy (Ed.). Springer International Publishing, Cham, 178-188.

[8] Wim De Mulder, Steven Bethard, and Marie-Francine Moens. 2015. A survey on the application of recurrent neural networks to statistical language modeling. Computer Speech and Language 30, 1 (2015), 61-98. https://doi.org/10.1016/j.csl. 2014.09.005

[9] Alex Graves, Abdel-rahman Mohamed, and Geoffrey Hinton. 2013. Speech recognition with deep recurrent neural networks. In 2013 IEEE International Conference on Acoustics, Speech and Signal Processing. IEEE, Piscataway, 66456649. https://doi.org/10.1109/ICASSP.2013.6638947

[10] M.A. Hannan, M.S.H. Lipu, A. Hussain, and A. Mohamed. 2017. A review of lithium-ion battery state of charge estimation and management system in electric vehicle applications: Challenges and recommendations. Renewable and Sustainable Energy Reviews 78 (2017), 834-854. https://doi.org/10.1016/j.rser.2017.05.001

[11] Sepp Hochreiter and Jürgen Schmidhuber. 1997. Long Short-Term Memory. Neural Computation 9, 8 (1997), 1735-1780. https://doi.org/10.1162/neco.1997.9. 8.1735

[12] Dickson N. T. How, M. A. Hannan, M. S. Hossain Lipu, and Pin Jern Ker. 2019. State of Charge Estimation for Lithium-Ion Batteries Using Model-Based and Data-Driven Methods: A Review. IEEE Access 7 (2019), 136116-136136. https: //doi.org/10.1109/ACCESS.2019.2942213

[13] Peter J. Huber. 1992. Robust Estimation of a Location Parameter. Springer New York, New York, NY, 492-518. https://doi.org/10.1007/978-1-4612-4380-9_35

[14] Asifullah Khan, Anabia Sohail, Umme Zahoora, and Aqsa Saeed Qureshi. 2020. A survey of the recent architectures of deep convolutional neural networks. Artificial Intelligence Review 53, 8 (2020), 5455-5516.

[15] Diederik P. Kingma and Jimmy Ba. 2015. Adam: A Method for Stochastic Optimization. In 3rd International Conference on Learning Representations, Yoshua Bengio and Yann LeCun (Eds.). ICLR, San Diego, 1-15. http://arxiv.org/abs/1412.6980

[16] Günter Klambauer, Thomas Unterthiner, Andreas Mayr, and Sepp Hochreiter. 2017. Self-Normalizing Neural Networks. In Proceedings of the 31st International Conference on Neural Information Processing Systems (Long Beach, California, USA) (NIPS'17). Curran Associates Inc., Red Hook, NY, USA, 972-981.

[17] Phillip Kollmeyer. 2018. Panasonic 18650PF Li-ion Battery Data. https://doi.org/ 10.17632/wykht8y7tg.1

[18] Phillip Kollmeyer, Carlos Vidal, Mina Naguib, and Michael Skells. 2020. LG 18650HG2 Li-ion Battery Data and Example Deep Neural Network xEV SOC Estimator Script. https://doi.org/10.17632/cp3473x7xv.3

[19] Reiner Korthauer. 2018. Lithium-ion batteries: basics and applications. Springer, Berlin.

[20] Shuai Ma, Modi Jiang, Peng Tao, Chengyi Song, Jianbo Wu, Jun Wang, Tao Deng, and Wen Shang. 2018. Temperature effect and thermal impact in lithium-ion batteries: A review. Progress in Natural Science: Materials International 28, 6 (2018), 653-666. https://doi.org/10.1016/j.pnsc.2018.11.002

[21] Dongxu Ouyang, Mingyi Chen, Jiahao Liu, Ruichao Wei, Jingwen Weng, and Jian Wang. 2018. Investigation of a commercial lithium-ion battery under overcharge/over-discharge failure conditions. RSC advances 8, 58 (2018), 3341433424 .

[22] Gianfranco Pistoia. 2014. Lithium-ion batteries: advances and applications. Elsevier Science, Amsterdam.

[23] Gregory L. Plett. 2004. Extended Kalman filtering for battery management systems of LiPB-based HEV battery packs: Part 2. Modeling and identification. Fournal of Power Sources 134, 2 (2004), 262-276. https://doi.org/10.1016/j.jpowsour. 2004.02.032

[24] Gregory L Plett. 2015. Battery management systems, Volume II: Equivalent-circuit methods. Artech House, United States.

[25] Juan Pablo Rivera-Barrera, Nicolás Muñoz-Galeano, and Henry Omar SarmientoMaldonado. 2017. SoC estimation for lithium-ion batteries: Review and future challenges. Electronics 6, 4 (2017), 102.

[26] Xiangbao Song, Fangfang Yang, Dong Wang, and Kwok-Leung Tsui. 2019. Combined CNN-LSTM Network for State-of-Charge Estimation of Lithium-Ion Batteries. IEEE Access 7 (2019), 88894-88902. https://doi.org/10.1109/ACCESS.2019. 2926517

[27] Carlos Vidal, Pawel Malysz, Phillip Kollmeyer, and Ali Emadi. 2020. Machine Learning Applied to Electrified Vehicle Battery State of Charge and State of Health Estimation: State-of-the-Art. IEEE Access 8 (2020), 52796-52814. https: //doi.org/10.1109/ACCESS.2020.2980961

[28] Yidan Xu, Minghui Hu, Anjian Zhou, Yunxiao Li, Shuxian Li, Chunyun Fu, and Changchao Gong. 2020. State of charge estimation for lithium-ion batteries based on adaptive dual Kalman filter. Applied Mathematical Modelling 77 (2020), 1255-1272. https://doi.org/10.1016/j.apm.2019.09.011

[29] Fangfang Yang, Xiangbao Song, Fan Xu, and Kwok-Leung Tsui. 2019. Stateof-Charge Estimation of Lithium-Ion Batteries via Long Short-Term Memory Network. IEEE Access 7 (2019), 53792-53799. https://doi.org/10.1109/ACCESS. 2019.2912803

[30] Wenpeng Yin, Katharina Kann, Mo Yu, and Hinrich Schütze. 2017. Comparative study of $\mathrm{CNN}$ and $\mathrm{RNN}$ for natural language processing. arXiv preprint abs/1702.01923 (2017). 